\title{
Efficiency Assessment for Indoor Microwave Wireless Power Transmission Systems
}

\section{Sohrab Bahrampour}

\author{
Submitted to the \\ Institute of Graduate Studies and Research \\ in partial fulfillment of the requirement for the Degree of
}

Master of Science

in

Electrical and Electronic Engineering

Eastern Mediterranean University

February 2015

Gazimağusa, North Cyprus 
Approval of the Institute of Graduate Studies and Research

Prof. Dr. Serhan Çiftçioğlu

Acting Director

I certify that this thesis satisfies the requirements as a thesis for the degree of Master of Science in Electrical and Electronic Engineering.

Prof. Dr. Hasan Demirel

Chair, The department of Electrical and Electronic Engineering

We certify that we have read this thesis and that in our opinion, it is fully adequate in scope and quality as a thesis for the degree of Master of Science in Electrical and Electronic Engineering.

Prof. Dr. Hasan Amca

Supervisor

Examining Committee

1. Prof. Dr. Hasan Amca

2. Assoc. Prof. Dr. Ali Hakan Ulusoy

3. Asst. Prof. Dr. Suna Bolat 


\begin{abstract}
Keeping the power hungry, smart wireless communication devices up and running became a challenging issue due to the need for continuous supply of battery power required for fulfilling all the functions. Since the battery capacity is limited, it needs to be recharged as often as possible. Hence, rather than spreading wires all around, it is a promising solution to cover the medium with electricity wirelessly or create charging points around residential premises based on Wireless Power Transmission technology. The work presented here involves investigation of the possibility of providing wireless charging points within a room and analyzing the choice of best frequency and best pulse shape for transmitting power wirelessly within a distance smaller than $10 \mathrm{~m}$ in order to charge batteries of such devices as smart phones. The parameters that can be adjusted to maximize efficiency of wireless power transmission and the relationship between the choice of frequency, pulse shape and distance is investigated. Antenna design will be left as a subject for future study.
\end{abstract}

Keywords: Microwave, Wireless, Power, Transmission, International Telecommunication Unity (ITU) model, Indoor, Propagation, Smart phone, Battery 
Akı1lı kablosuz iletişim araçlarının sorunsuz çalışır durumda tutmak, bütün işlevlerini yerine getirmeleri için gerekli sürekli enerji ihtiyaçları nedeniyle, zorlu bir konu haline gelmiştir. Pil kapasite sınırlı olduğu için bu tür cihazlar sık sık şarj edilmelidir. Her yere kablo döşemek yerine; kablosuz güz iletimi teknolojisiyle ortamı kablosuz şekilde elektriklendirmek veya evler içinde kablosuz şarj etme noktaları oluşturmak gelecek vaad eden bir çözüm olabilir. Bu çalışmada bir oda içinde akıllı telefonlar gibi araçların pillerini şarj etmek için kablosuz şarj etme noktaları oluşturma olasılığı araştırılmış ve 10 m’den az uzaklığa kablosuz güç iletimi için en iyi frekans ve dalga şekli analiz edilmiştir. Kablosuz güç iletiminin verimini arttırmak için ayarlanan parametreler; frekans seçimi, dalga şekli ve mesafe arasındaki ilişki araştırılmıştır. Anten tasarımı gelecek çalışmaları için bir konu olarak bırakılmıştır.

Anahtar Kelimeler: Mikrodalga, Kablosuz, Enerji, İletim, İTÜ modeli, İç mekan, Yayılım, Akıllı telefon, Pil 
This research is dedicated to my lovely mother and sister for their continued support and encouragement during my whole life. 


\section{ACKNOWLEDGMENT}

I would like to express my special thanks of gratitude to my supervisor Prof. Dr. Hasan Amca for all his help and support in every steps of this master thesis process. I would like to thank him for encouraging my research and for allowing me to grow as a research scientist.

I would also like to thank my committee members, Professor Ali Hakan Ulusoy and Professor Suna Bolat for serving as my committee members even at hardship. I would also like to thank them for letting my defense be an enjoyable moment and for all their brilliant comments and suggestions.

Finally the last, but not the least, I would like to thank my family who have always supported me during my life. Words cannot express how grateful I am to my mother and sister for all of the sacrifices that they have made on my behalf. Their prayer for me was what sustained me thus far. 


\section{TABLE OF CONTENTS}

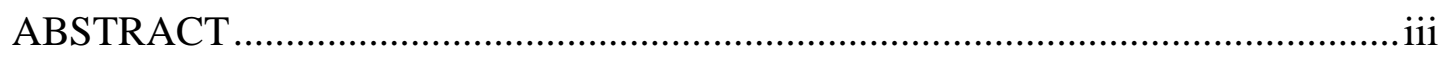

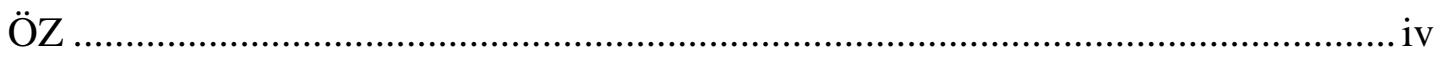

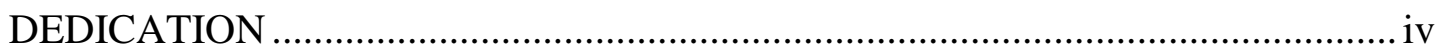

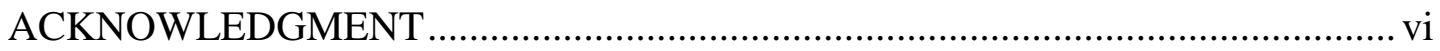

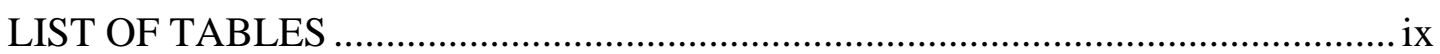

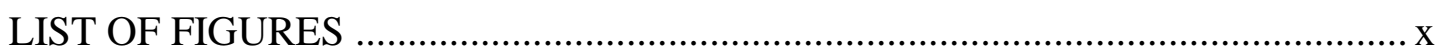

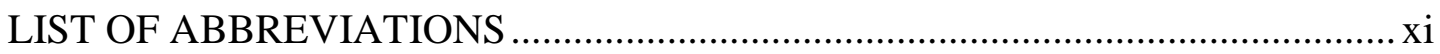

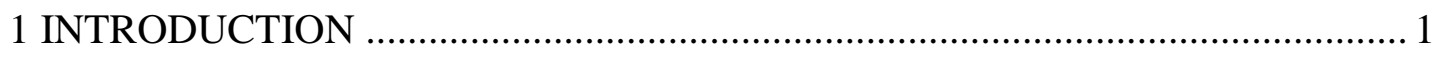

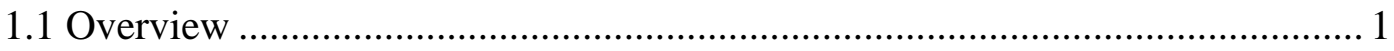

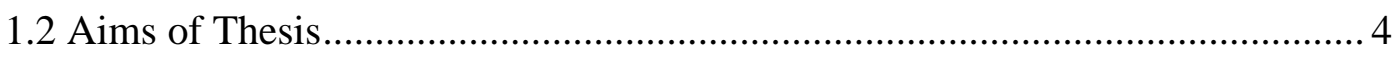

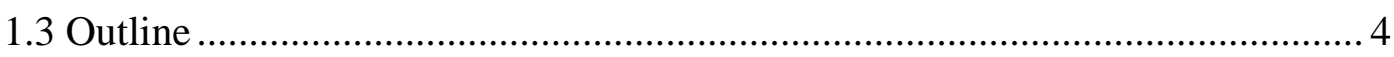

2 REVIEW OF WIRELESS POWER TRANSMISSION ...................................... 5

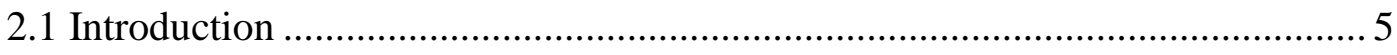

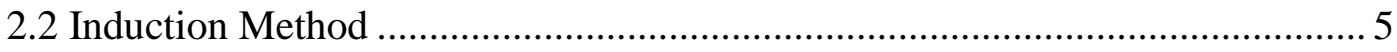

2.2.1 Non-Resonant Induction Method........................................................ 6

2.2.2 Resonant Induction Method ................................................................. 7

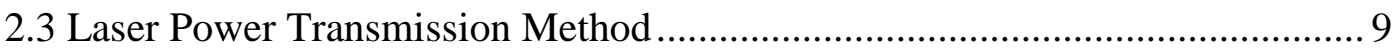

2.4 Microwave Wireless Power Transmission Method........................................ 11

3 WIRELESS POWER TRASMISSION CHANNEL CHARACTERISTICS ......... 14

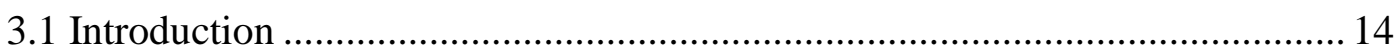

3.2 General Characteristics of Indoor Transmission Medium.............................. 15

3.3 Path Loss Model Due to Multipath Propagation in Indoor Transmission Medium 
3.4 The Use of Narrow-Beam Directional Antenna ........................................... 21

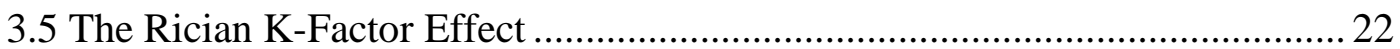

4 MICROWAVE WIRELESS POWER TRANSIMISSION SYSTEM MODELING

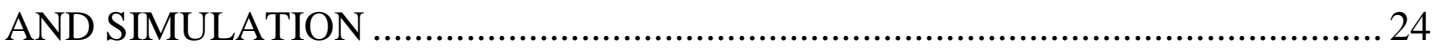

5 CONCLUSION AND RECOMMENDATIONS FOR FURTHER STUDIES ...... 34

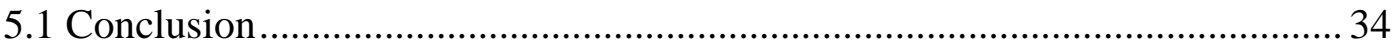

5.2 Recommendations for Future Works ........................................................... 35 


\section{LIST OF TABLES}

Table 3.1: Comparison of the features of WDC and MW-WPT in an indoor

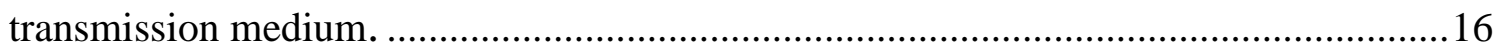

Table 3.2: Distance Power Loss Coefficient factor for Indoor Transmission .................20 


\section{LIST OF FIGURES}

Figure 2.1: Basic principles of Induction Method [16] ........................................ 7

Figure 2.2: Tesla induction charging in CES 2012 ........................................... 8

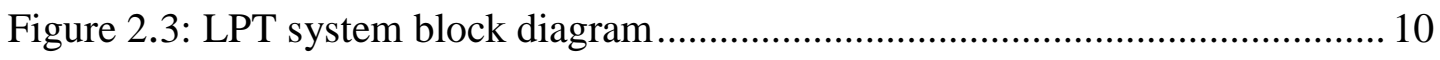

Figure 2.4: Test-Bed for MW-WPT over indoor transmission medium.................... 12

Figure 3.1: The multipath propagation model consists of a LOS and relatively low power reflections.

Figure 4.1: $P R x$ versus distance $(d)$ graph for different frequencies of operation for basic rectangular pulse shape.

Figure 4.2: $P R x$ versus distance $(d)$ graph for different frequencies of operation comparison between raised cosine pulse shape and rectangular pulse shape. 28

Figure 4.3: $P R x$ versus distance $(d)$ for LOS and non-LOS (NLOS) cases of rectangular pulse shape.

Figure 4.4: $P R x$ versus distance $(d)$ for different Rician K-Factors of rectangular pulse shape with $2.5 \mathrm{GHz}$ carrier frequency. 33 


\section{LIST OF ABBREVIATIONS}

\begin{tabular}{|c|c|}
\hline $\mathrm{AC}$ & Alternative Current \\
\hline CES & Consumer Electronics Show \\
\hline DC & Direct Current \\
\hline $\mathrm{E} / \mathrm{O}$ & Electrical to Optical \\
\hline $\mathrm{EV}$ & Electric Vehicles \\
\hline FF-WPT & Far Field Wireless Power Transmission \\
\hline $\mathrm{HEV}$ & Hybrid Electric Vehicles \\
\hline IM & Induction Method \\
\hline ISM & Industrial, Scientific and Medical \\
\hline ITU & International Telecommunication Union \\
\hline JPL & Jet Propulsion Laboratory \\
\hline LB-WPT & Laser Beam Wireless Power Transmission \\
\hline LD & Laser Diode \\
\hline LPT & Laser Power Transmission \\
\hline LTSP & Latest Technology Smart Phone \\
\hline LOS & Line Of Sight \\
\hline MW-WPT & Microwave Wireless Power Transmission \\
\hline
\end{tabular}


NASA

NF-WPT

NRIM

$\mathrm{O} / \mathrm{E}$

PVC

$\mathrm{RF}$

RFID

RIM

WPT
National Aeronautics and Space Administration

Near Field Wireless Power Transmission

Non Resonant Induction Method

Optical to Electrical

Photo-Voltaic-Cell

Radio Frequency

Radio Frequency Identification

Resonant Induction Method

Wireless Power Transmission 


\section{Chapter 1}

\section{INTRODUCTION}

\subsection{Overview}

Back to early 1819s, when Hans Christian Orsted and Andre Marie Ampere recognized electromagnetism and the unity of electric and magnetic phenomena, people started to get familiar with electricity. Ever since, electricity has been extensively used by humans in illumination, operating machines and finally distant communication to improve the quality of human's life. Eventually, the electric power's demand increased by people. All these changes led scientists to create a way to transmit electricity from where it is generated, to domestic and industrial premises where the electric power is consumed.

In 1882, the first transmission line based on Direct Current (DC) transmission method was designed. However, due to the variety of load demanded and power losses during transmission, the DC transmission method was found not to be efficient. Later on, in 1886 the first $1 \mathrm{kV}$ Alternating Current (AC) transmission line was introduced in Great Barrington, Massachusetts. It was the beginning of the present power transmission system [1].

As the time went on, technological developments brought a large number of electrical and electronic devices to the human life and the need of energizing these 
devices becomes an important issue for users. Devices served with batteries or those plugged in directly to electricity need an always available electricity source to fulfill all their functions. These demands resulted in producing cables and batteries for delivering power to the electronic devices. These cables are made of aluminum and copper conductors, which have limited resources and are expensive. On the other hand, as the number of electronic devices in a place increases, too many cables bring a messy situation which can cause many problems. Therefore, to handle these difficulties, a new method of energizing electronic devices needed to be introduced. In this work, we are concentrating on Wireless Power Transmission (WPT) method as a solution to the mentioned problems. WPT is transmitting electricity without using man-made conductors and was first introduced by Nikola Tesla in the early 19th century [2]. His experiments were a great step toward a new generation of electricity transmission. Although his works did not achieve an appropriate result because of low efficiency, it was a beginning for scientists and researchers to find a more efficient way for WPT. There are three different types of WPT techniques in the technological literature, namely, Laser Beam Wireless Power Transmission (LBWPT), Near Field Wireless Power Transmission (NF-WPT) and Far Field Wireless Power Transmission (FF-WPT).

Due to the characteristics of the laser beam, the LB-WPT method needs a Line of Sight (LOS) between the transmitter and the receiver in order to enable the laser beam produce electricity at the photocell receiver. National Aeronautics and Space Administration (NASA) has done an experiment to energize an unmanned aircraft by 
lighting a laser beam to a photocell plate installed on the aircraft in 2003 [3]. NASA is currently working on the project of energizing satellites from earth using any suitable WPT technology. The NF-WPT is mostly concentrated on magnetic induction method [4-6] which are based on LC circuits and inductive resonance. The efficiency of NF-WPT is very high due to the short distance between the transmitter and the receiver coil. The shorter the distance, the higher the efficiency. The FFWPT method is based on transmitting and receiving Radio Frequency (RF) signals at a certain distance.

One of the first experiments using this method was performed in World War II during the development of cavity magnetron [7]. Later on, interesting experiments were carried out at Raytheon's Spencer Laboratory in May 1963 using Microwave Wireless Power Transmission (MW-WPT) technique with an efficiency of $13 \%$ to drive a 100W DC motor [8]. In search of the best frequency for WPT, in 1975 Goldstone designed an experiment using a $26 \mathrm{~m}$ diameter parabolic transmitter antenna using $2.388 \mathrm{GHz}$ frequency. Within a $1.54 \mathrm{~km}$ distance from transmitter, the $3.4 \times 7.2 \mathrm{~m}^{2}$ rectenna could just achieve $6.7 \%$ efficiency [9]. The transmission efficiency in this experiment was not satisfying, despite the impractically high dimensions of the transmitter and receiver rectenna. Developing rectennas to achieve the best efficiency, researchers made efficiency improvements during 1992-1994, concentrating on 2.45 and $35 \mathrm{GHz}$ [9-12]. In 2000, at the Jet Propulsion Laboratory (JPL), based on the availability of the high power X-band sources an experiment of 8.51 GHz was held [13]. Most of the works done in MW-WPT have concentrated on 
the $2.450 \mathrm{GHz}$ Industrial, Scientific and Medical (ISM) band frequency due to its power transmission efficiency and relatively lower human health concerns [7][1415]. The WPT system efficiency can be improved by transmitting a specially designed signal such that the LOS and reflections will add constructively at the receiver. The focus of this thesis is on the choice of the shape and characteristics of the transmitted signal in order to maximize the received power at the rectenna output.

\subsection{Aims of Thesis}

In this thesis, we are concentrating on the effect of the transmission channel characteristics. Our goal is to design a system to charge a battery of a typical smart phone. Our focus is on indoor WPT within distances smaller than $10 \mathrm{~m}$.

\subsection{Outline}

The thesis is organized as follows: In Chapter 1, an introduction to WPT is given. In Chapter 2, the various WPT techniques are reviewed and Chapter 3 concentrates on the choice of best frequency and transmitted pulse shape for maximizing WPT system transmission efficiency. WPT Channel Modeling in Chapter 4 is followed by Simulation Results and Conclusions in Chapter 5 and 6. 


\section{Chapter 2}

\section{REVIEW OF WIRELESS POWER TRANSMISSION}

\subsection{Introduction}

In this work, WPT is referred to as the concept of energizing electrical devices without using conductor wires. Generally WPT is based on three different methods. Induction method which is based on principles of magnetic fields and is mostly useful among close distances. The next solution to the WPT is laser power transmission. This method is well known for energizing immobile devices in far distances. The third method is microwave WPT which is expected to be the solution to the best efficiency of WPT system. An overview of these three methods is presented in this chapter.

\subsection{Induction Method}

One of the most famous WPT methods in research is Induction Method (IM). In the IM, the magnetic field produced by the transmitter coil induces a magnetic field at the receiver coil, which in turn produces a current when the receiver coil is located in the transmitter coil's magnetic field. Based on the characteristics of the IM, magnetic field can feed the receiver coil in two different ways; Non-Resonant Induction Method (NRIM) and Resonant Induction Method (RIM). 


\subsubsection{Non-Resonant Induction Method}

NRIM is useful over short distances such as less than a few centimeters when the transmitter and receiver are aligned. For this purpose, the transmitter coil is placed in a plate and the receiver coil is then located inside the device, connected to the battery's circuit. Laying the device on the transmitter plate will charge the device's battery. When the distance between transmitter and receiver is close to zero, the NRIM has maximum performance and by increasing the distance till few centimeters, depends on the transmitter coil's characteristics; the amount of transmitted power will quickly drop to zero. This method is mostly useful for charging devices like smart phones, laptops, tablets and etc. For the best efficiency in NRIM, the transmitter coil and the receiver coil should be as close as possible. So one may easily put his laptop or smart phone simultaneously on the table and work with that. Intel and Samsung Companies are using induction charging method for some models of their laptops since 2013. Also some companies like Nokia (for the Lumia 820 and Lumia 920), Google-LG (for the Nexus 4, Nexus 5 and Nexus 7), Motorola Mobility (for the Droid 3 and Droid 4), HTC (for the Droid DNA), Samsung (for the Galaxy S4) and Apple (for I Watch) are using NRIM for their products since 2012. Utilization of NRIM technology is growing sharply and the efficiency of the system is improving, as well. For clarifying the whole idea of the system, Figure 2.1 simply demonstrates the basics of NRIM. 


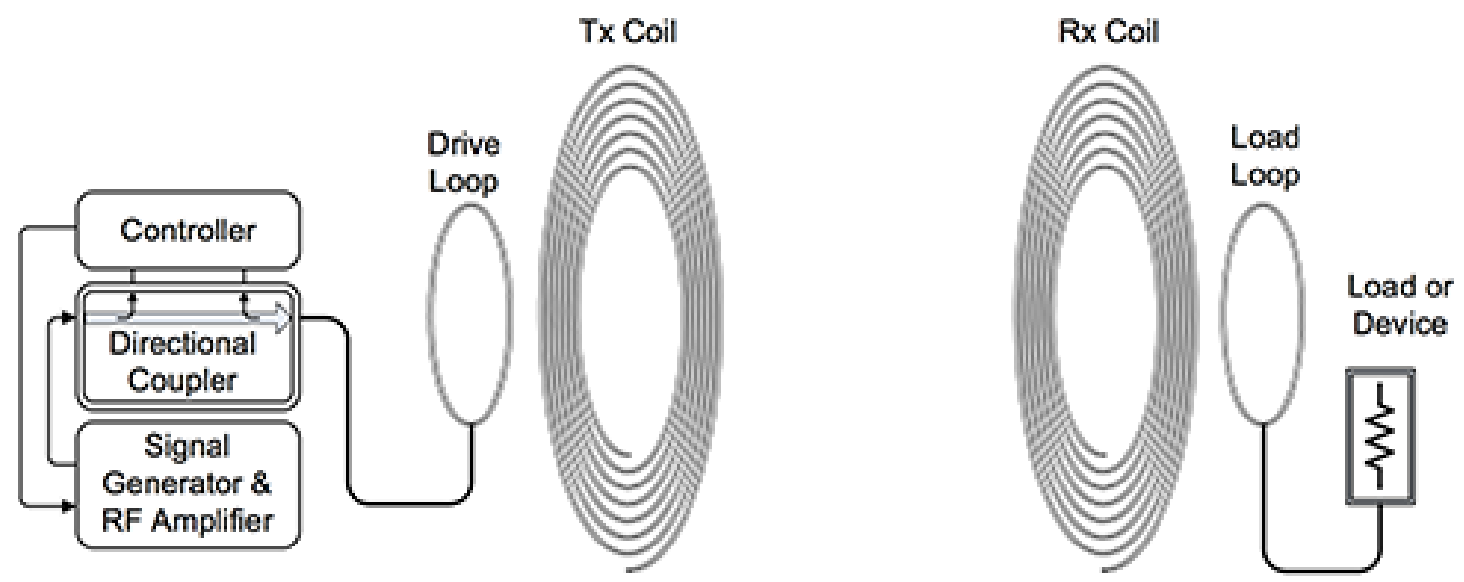

Figure 2.1: Basic principles of induction method [16]

If the distance of transmission becomes larger than few centimeters, NRIM is not practical anymore. For larger distances the RIM is a better solution.

\subsubsection{Resonant Induction Method}

In this method, transmitter and receiver coils are tuned to resonate at the same frequency. So, the transmitter coil can serve larger distances compared to NRIM but yet not enough far. To improve the efficiency of NRIM, tunneling is applied to the magnetic field between transmitter and receiver. Although larger distances are covered using this method, the largest distance, considering careful alignment of transmitter and receiver coils, is less than 2 meters. NRIM is a common technology used from years ago in Radio Frequency Identification (RFID) tags. Recently, WiTricity and Rezence companies are doing so many researches on IM to produce transmitter plates which can serve different devices with different demands and they have made remarkable achievements in this area. One of the most valuable achievements in this field belongs to the production of Hybrid Electric Vehicles (HEVs) and Electric Vehicles (EVs) where induction charging methods are used to charge vehicle's batteries. Among all these companies, Tesla Motors is a pioneer 
company producing EVs and electric power trains components like lithium-ion batteries and wireless chargers. Figure 2.2 shows a wireless charger based on induction method used for Tesla Roadster car presented in Consumer Electronics Show (CES) 2012. Recently, different companies are working on wireless charging pathways to charge EV's batteries while the vehicle is moving.

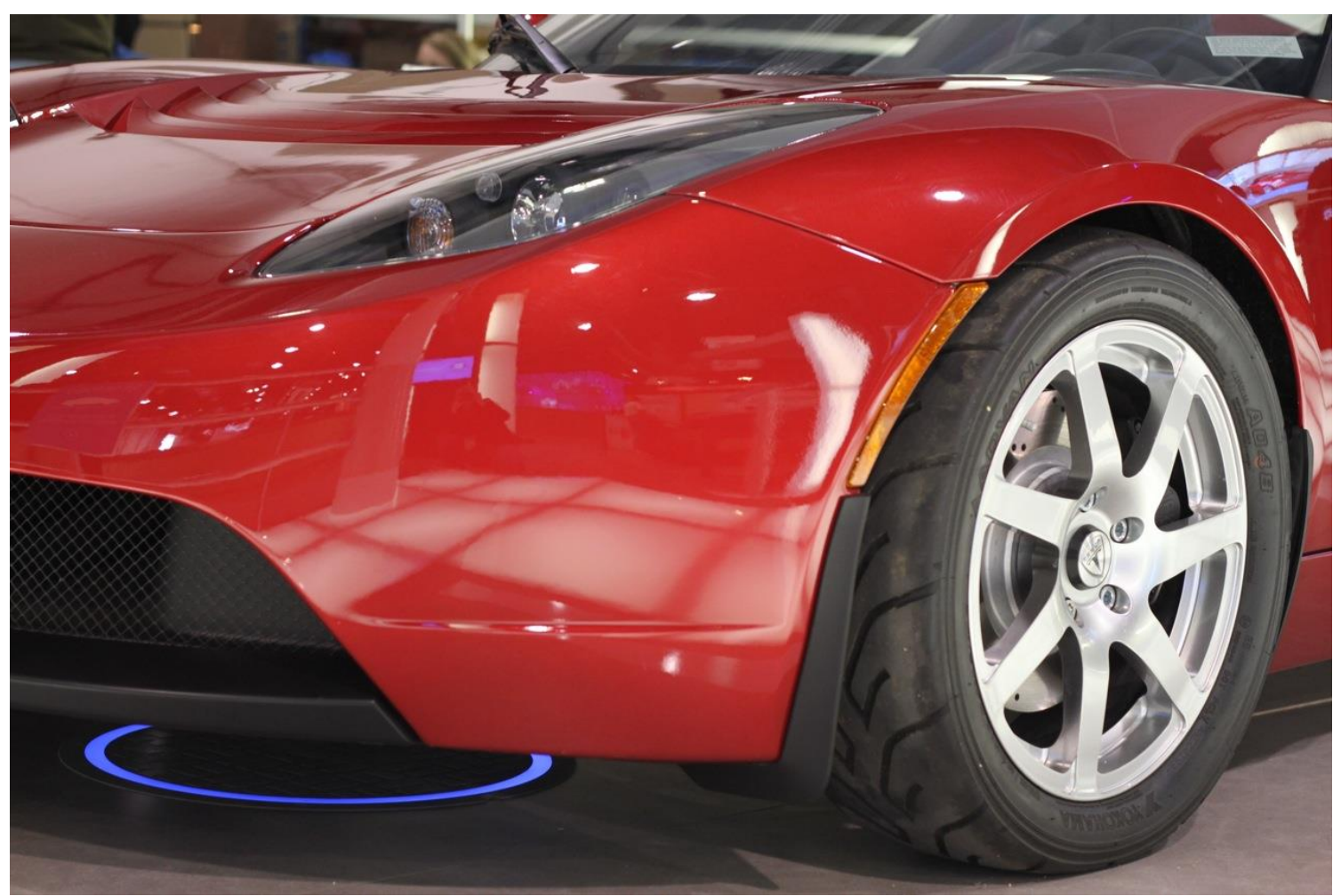

Figure 2.2: Tesla induction charging in CES 2012

According to the magnetic field properties, IM cannot be useful over long distances because the transmitter coil will need huge amount of power to produce a strong magnetic field at the receiver coil and the size of the coil will get extremely large. On the other hand, human health concerns will become significantly important under such powerful magnetic field conditions as it can ionize human's blood and result in death. 
This lack of ability for the IM to transmit power wirelessly to larger distances leaded scientists to search for other methods such as Laser Power Transmission (LPT) and MW-WPT.

\subsection{Laser Power Transmission Method}

The LPT method was introduced to energize devices at more distant points. Here, the laser beam is projected on a specially designed Photo-Voltaic-Cell (PVC) which is placed on the receiver consumer. The receiver will convert the optic energy of the laser beam into electric current. Total efficiency of LPT system completely depends on efficiency of Optical-to-Electrical (O/E) and Electrical-to-Optical (E/O) units. In other word, $\mathrm{O} / \mathrm{E}$ is the PVC part that acts as the receiver and $\mathrm{E} / \mathrm{O}$ is the Laser Diode (LD) where it works as the transmitter. According to human health hazards, safe operating wavelength for power beaming is specified by the IEC 60825-1 standard [17]. This safety standard is calculated by dividing optical power in watts, over the unit area in square meters where it is called the power density of the laser beam. Generally, if the wavelength is long and duration of laser exposure is short, it is safe to operate the system based on IEC 60825-1 standard.

However, while solving the problem about transmitting power wirelessly to relatively larger distances, the necessity for a clear LOS created a weak point in the LPT method. In addition, exact positioning and directionality of the LPT method still poses a serious problem [18]. A simple block diagram of the LPT system is presented in Figure 2.3 in order to interpret the basics. 


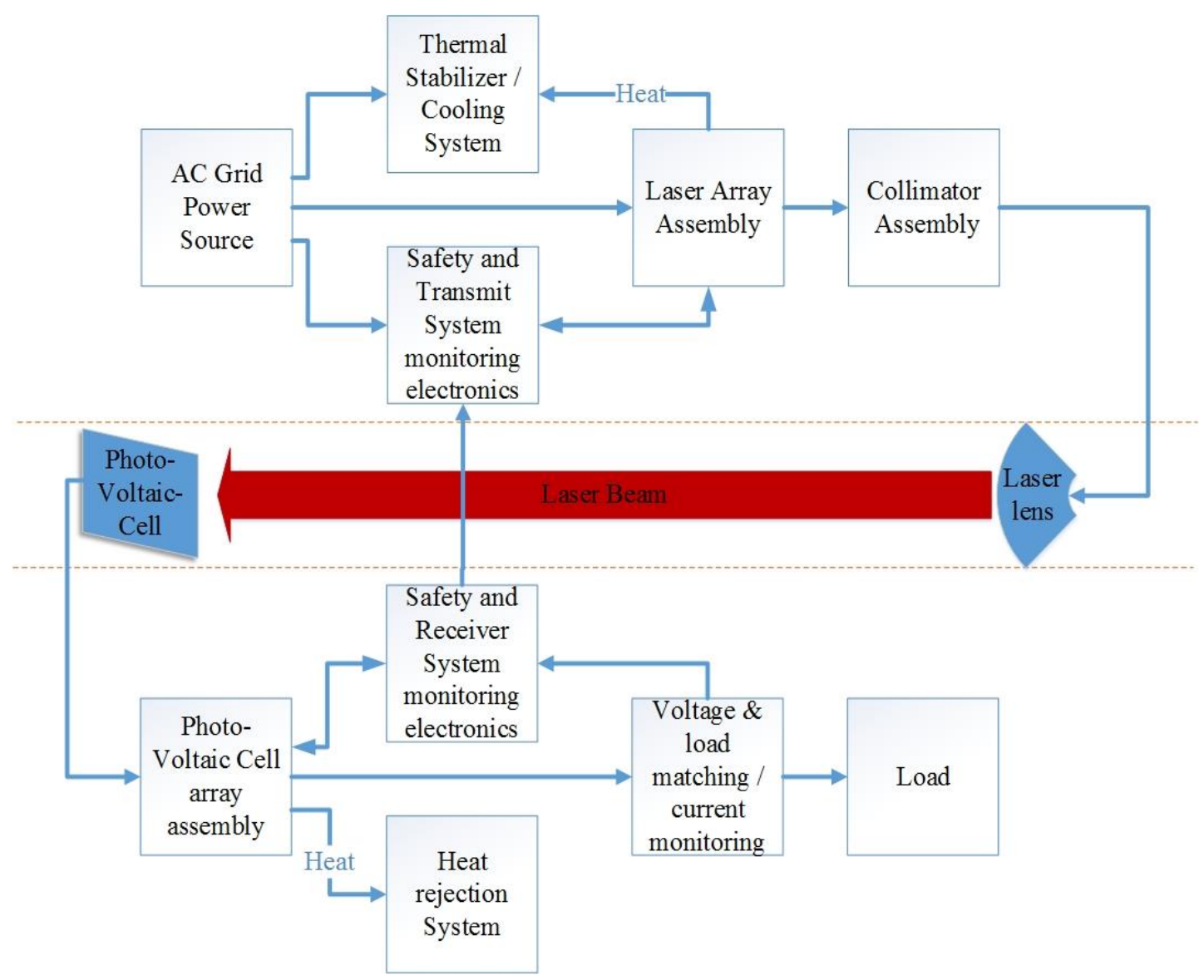

Figure 2.3: LPT system block diagram

The AC power source is used to energize controlling and monitoring devices of the transmitter part, also to assemble the laser array. Monitoring and controlling units of the transmitter will check the safety of the system in order to protect the unit against over heat and power of the laser beam. The laser array is then followed by a collimator to produce a collimated light beam to produce the maximum overlap on the active region of the photo voltaic with the free space in between. The laser is projected to the photo voltaic cell by the laser lens. Photo voltaic cell collects the laser beam and converts the optical power into electrical power. A heat rejection system is the placed in the receiver part to remove the over heat of the system. Receiver safety monitoring unit controls the received power to report any possible 
faults of the transmission. This electrical power then goes through a load matching unit to set the power based on the load demand.

\subsection{Microwave Wireless Power Transmission Method}

The next alternative solution for WPT is the MW-WPT method where the RF signal in microwave frequency range is produced in the transmitter antenna and transmitted in the air. Coverage of the MW-WPT method can be adoptive depending on the transmitter power and the antenna configuration. The receiver is simply consist of a combination of an antenna and a rectifying circuit, called rectenna in order to collect the microwave energy and produce electricity. The use of MW-WPT method is expected to solve many problems related to WPT. However, the efficiency of the system is still unacceptably low, that poses a big problem for practical applications. MW-WPT method is known as one of the best solutions for WPT due to its capacity to transmit power to long distances, and its tolerance to miss-alignment of the transmitter and receiver. In MW-WPT method, the transmitter antenna, medium between transmitter and receiver, channel characteristics and the configuration of rectenna are effective elements of system total efficiency. Transmitter and receiver antennas can be considered whether as omnidirectional or directional. Because of greater concentration of radiation in a specific direction, the performance of directional antennas is higher compared to omnidirectional antennas. But as directionality is a weak point in WPT systems, omnidirectional antennas are chosen in MW-WPT method. Then the system's efficiency just depends on medium and channel characteristics whereas generally the medium is air, so the only factor remains is channel characteristics. 
In this work, we are concentrating on improving efficiency and coverage flexibility of MW-WPT method with reference to the block diagram presented in Figure 2.4.

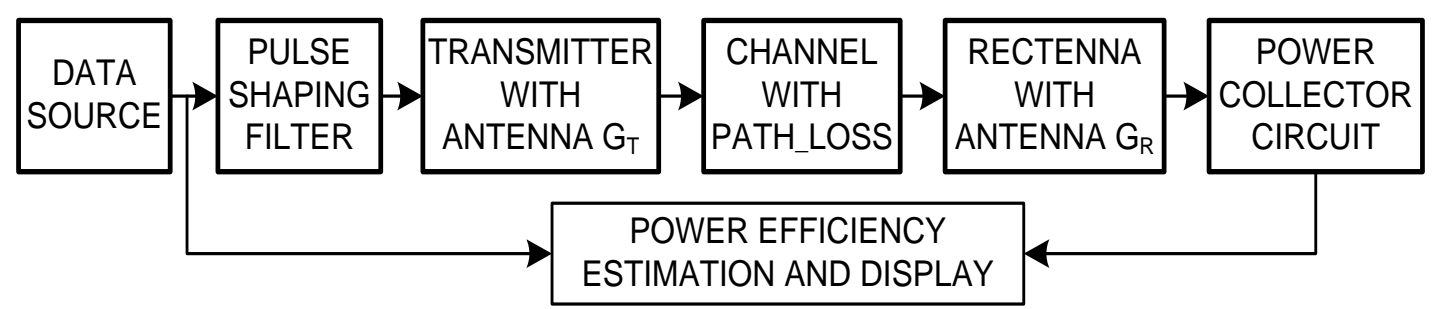

Figure 2.4: Test-Bed for MW-WPT over indoor transmission medium

In Figure 2.4, the data source generates an AC signal. The AC signal is produced arbitrary using a Bernoulli Binary algorithm as a combination of ones and zeroes. These values will then go through a pulse shaping filter. Pulse shaping filters used in this work are rectangular and raised-cosine in order to determine whether the shape of transmitted signal is significant for the power received in the rectenna or not.

The channel in Figure 2.4 is considered as Rician and Rayleigh fading to compare different results of the test. Rician fading channel is used for the situations where a direct LOS is available between transmitter and receiver antennas. There may also be some periods of time which the LOS is blocked because of obstacles or moving objects. The Rayleigh fading channel is used for the time where there is no LOS available in the medium. More details of the channel parameters will be discussed in the next section.

The receiver part is made of an antenna and a rectifier circuit. The aggregation of these two is called rectenna. Rectenna works as a signal collector and it converts the 
RF signal into DC power. Therefore the choice of rectenna is also a critical issue for maximizing transmission efficiency.

The MW-WPT method employed in this work is used to charge electronic devices such as a Latest-Technology Smart Phone (LTSP). A LTSP such as an IPhone 5s typically has $1650 \mathrm{mAh}$ battery. The minimum energy source required to charge such a battery is $2.5 \mathrm{~W}$ with a minimum $0.5 \mathrm{~A}$ current and $5 \mathrm{~V}$ DC voltages. Hence, this work concentrates on producing such amount of power in the receiver part, so that the targeted devices are charged. Generally, because of transmission power losses, rectenna size and other factors such as human health hazards, it is not possible to have a 2.5 Watts power at the receiver.

In order to produce the required amount of power we need to employ voltage multiplier circuit after the power collector circuit shown in the Figure 2.4. A voltage multiplier circuit typically uses a network of diodes and capacitors to increase the voltage level in the terminals. There are different kinds of voltage multipliers available but one of the best choices for voltage multiplier circuit is Dickson DC-DC charge pump which can make the output voltage up to five times bigger [19]. Generally it will take about 40 minutes to fully charge an IPhone 5s's battery using a suitable charge pump. 


\section{Chapter 3}

\section{WIRELESS POWER TRASMISSION CHANNEL CHARACTERISTICS}

\subsection{Introduction}

According to what have passed, there are too many factors in a WPT system which can affect the total efficiency of the system. Among all these elements the channel characteristics of the system has one of the most ruinous roles in this scenario. The major impairments of an indoor wireless channel are specular reflections from the smooth walls, ceilings and floors and the thermal noise of the medium.

Refraction's influence occurs when the signal passes through different materials. In fact, when the signal passes from one medium to another, as the direction of the signal changes, degradation in signal strength may happen. Also, if the system is designed as a point to point link, the communication may be lost because of the signal deflection. The transmitted signal in a WPT system may also undergo diffraction. The receiver device of the WPT system may sometimes shadowed by an obstacle and the signal will reach receiver by diffraction. The diffraction effect is completely depended on the size of obstacle and the wavelength and based on these factors, so the power strength received in the receiver may decrease sharply. Generally, if the frequency is low, it can diffract around larger obstacles and vice 
versa. Scattering on the other hand is an important factor, as well. The received power in the receiver is always more than summation of reflection and diffraction. This amount of power is because of the effect of scattering. When a radio wave propagates on a ragged surface, the reflection of the wave is diffused in all directions. This phenomenon is called scattering and the energy from these diffused waves can be collected in the receiver to improve the receive power.

In addition, there will be other transmission losses when waves travel through obstacles such as walls, floors and ceilings [20]. Hence, these impairments in the transmission medium could cause substantial amounts of time and/or space variant path loss which can result in significant reduction in signal strength and cause polarization problems. To find out about the best efficiency of the system, first we need to know about general characteristics of an indoor transmission medium.

\subsection{General Characteristics of Indoor Transmission Medium}

When modeling and analyzing the efficiency of MW-WPT techniques, the characteristics and behavior of the channel plays a major role. It is, therefore, important to compare and contrast the characteristics and behavior of the indoor transmission medium for usage in data transmission and power transmission distinctly, as stated in Table 3.1 below. 
Table 3.1: Comparison of the features of WDC and MW-WPT in an indoor transmission medium

\begin{tabular}{|l|l|}
\hline \multicolumn{1}{|c|}{$\begin{array}{c}\text { Wireless Data Communication } \\
\text { (WDC) }\end{array}$} & \multicolumn{1}{|c|}{$\begin{array}{c}\text { Microwave Wireless Power } \\
\text { Transmission (MW-WPT) }\end{array}$} \\
\hline $\begin{array}{l}\text { Despite the fact that channel quality } \\
\text { rarely falls below unacceptable levels, } \\
\text { this rare situation may result in very low } \\
\text { system performance. e.g., when the } \\
\text { channel experiences a deep fade due to } \\
\text { destructive addition of multipath } \\
\text { components, the resulting signal can be } \\
\text { useless for successful WDC. Therefore, } \\
\text { quality channel conditions does not } \\
\text { matter since only the cumulative } \\
\text { summation of instantaneous power } \\
\text { levels are of interest in MW-WPT. } \\
\text { Even when the channel is in a deep } \\
\text { fade, transmission efficiency will not be } \\
\text { conditions must be taken to avoid such } \\
\text { hindered significantly since the } \\
\text { occurrence of deep fades are very rare } \\
\text { in indoor transmission medium [20]. }\end{array}$ \\
$\begin{array}{l}\text { Usually, advanced channel estimation } \\
\text { and detection techniques rely on } \\
\text { detection of the amplitude and phase of } \\
\text { the received signal where most of the } \\
\text { computational power and time is } \\
\text { consumed. }\end{array}$ & $\begin{array}{l}\text { The cumulative summation of the } \\
\text { magnitude of the received signal is } \\
\text { sufficient for successful MW-WPT. } \\
\text { Hence, detection of the frequency and } \\
\text { phase is not necessary and thus the } \\
\text { detector can be very simple. }\end{array}$ \\
&
\end{tabular}

It can easily be judged from Table 3.1 that, the channel impairments such as multipath propagation, thermal noise, or phase noise that hinder the performance of indoor WDC does not have a severe negative effect on MW-WPT. Contrary to WDC, the power in the multipath components may improve systems efficiency in MW-WPT. This is depicted by the multipath propagation model in Figure 3.1 where contribution of the LOS and reflections to the composite multipath signal is shown. Beside the LOS, there are other important power sources in the MW-WPT system. As mentioned before, reflections from walls, ceilings and all objects in the indoor medium can be considered as a wave source for the receiver antenna. These rays may 
not be able to carry data over WDC system or to improve the system efficiency, but in MW-WPT we just need the power of the rays.

Each of the rays arriving at the receiver due to the transmitted signal will experience a different path loss and phase shift. Each of these path losses and phase shifts has its specific power and characteristic which will cause different sufficient improvement to the MW-WPT system's competence. The path loss due to multipath propagation in indoor transmission medium will be discussed in the following section where as the phase shift will be ignored due to its irrelevance for MW-WPT.

\subsection{Path Loss Model Due to Multipath Propagation in Indoor Transmission Medium}

The indoor transmission model used in this work is based on an indoor scenario. The distance between transmitter and receiver antennas is considered to be maximally 10 meters and both transmitter and receiver are supposed to be located in the same room. Therefore, for such indoor medium the transmission path loss contains two different source of energy. One is the LOS path loss which in fact brings the most effective signal strength to the receiver. The other is because of multiple reflections of the transmitted signal to the receiver antenna. Although these reflections are not as strong as LOS path loss, they have a substantial effect on the total effectiveness of the system. There are many path loss models available in the literature that account for attenuation through walls, floors and ceilings as well as shadowing. 


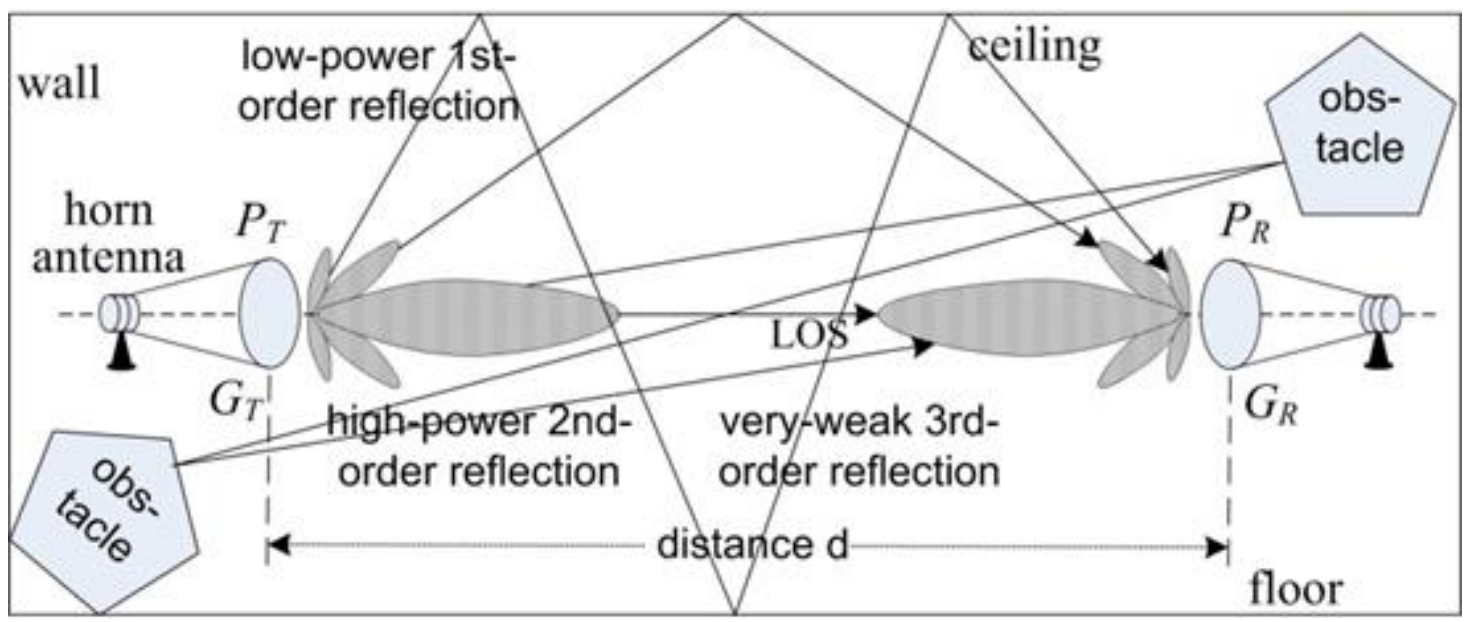

Figure 3.1: The multipath propagation model consists of a LOS and relatively low power reflections

For the MW-WPT path loss model, only signal behavior inside the same room is considered since the transmitter and receiver is placed in the same room and the reflections from neighboring rooms are negligibly weak. In the indoor propagation circumstances, the amount of coverage depends on the geometry of the building. Also there are specific restrictions in each building which modify the propagation. There is also a frequency reuse in an indoor model which can bring considerable effects to the propagation characteristics. Therefore because of intricate nature of these fundamentals, if a specific planning for an indoor system of a particular building is to be assumed, all detailed information of the area including geometry, furniture, materials and so on should be considered. Consequently, in order to study about MW-WPT system, we need to use a general model that generally represents the propagation characteristics of the area. On the other hand, the model should not need too much input data for the calculations. Hence, effects such as penetration through building materials will be eliminated from the general formula published in the literature. Among all different standards available for indoor propagation, 
International Telecommunication Union (ITU) has developed a useful model which is used in this study. The basic path loss model presented in the ITU-R P1238-7 recommendations at [21]can serve as the best model for MW-WPT as:

$$
P L(d B)=20 \log _{10}\left(f_{c}\right)+N \times \log _{10}(d)+L_{f}(n)+R
$$

where $N$ is the distance power loss coefficient, $f_{c}$ is the transmission frequency in $\mathrm{MHz}, d$ is the separation between the transmitter and receiver in meter, $L_{f}(n)$ is the wall, floor and ceiling penetration loss factor and nis number of walls, floors and ceilings between the transmitter and receiver and $R$ is the adaptation constant for different locations. For the case at hand while we consider that the transmitter and receiver are in the same room, $L_{f}(n)$ is equal to 0 and therefore considering the adaption constant $(R)$ for this study, these terms are dropped from the equation. So as a result, equation (1) will reduce to:

$$
P L(d B)=20 \log _{10}\left(f_{c}\right)+N \log _{10}(d)-28
$$

According to the ITU-R P1238-7 recommendation, the distance power loss coefficient differs and depends on which kind of indoor location and what frequency the transmitter propagates. Table 3.2 indicates different values of power loss coefficient, obtained from numerous measurement results. 
Table 3.2: Distance Power Loss Coefficient factor for Indoor Transmission

\begin{tabular}{|c|c|c|c|}
\hline Frequency & Residential & Office & Commercial \\
\hline $900 \mathrm{MHz}$ & - & 33 & 20 \\
\hline $1.2-1.3 \mathrm{GHz}$ & - & 32 & 22 \\
\hline $1.8-2 \mathrm{GHz}$ & 28 & 30 & 22 \\
\hline $2.4 \mathrm{GHz}$ & 28 & 30 & - \\
\hline $3.5 \mathrm{GHz}$ & - & 27 & - \\
\hline $4 \mathrm{GHz}$ & - & 28 & 22 \\
\hline $5.2 \mathrm{GHz}$ & $28-30$ & 31 & - \\
\hline $5.8 \mathrm{GHz}$ & - & 24 & - \\
\hline $60 \mathrm{GHz}$ & - & 22 & 17 \\
\hline $70 \mathrm{GHz}$ & - & 22 & - \\
\hline
\end{tabular}

Therefore if an office indoor environment with a $2.4 \mathrm{GHz}$ frequency is a selected example, then the equation (2) changes to:

$$
P L(d B)=20 \log _{10}\left(f_{c}\right)+30 \log _{10}(d)-28
$$

where the distance between the transmitter and receiver $d$ is in meter and the transmission frequency $f_{c}$ is in $\mathrm{MHz}$, hence for the sample transmission frequency $f_{c}=2400 \mathrm{MHz}$, equation (3) becomes:

$$
P L(d B)=20 \log _{10}(2400)+30 \log _{10}(d)-28
$$

Which then equation (4) turns to:

$$
P L(d B)=30 \log _{10}(d)+39.6
$$


The indoor channel model given by [22] has no frequency component as shown in (6). This model is not preferred since we are also interested in the behavior of the MW-WPT system at different frequencies.

$$
P L(d B)=37+30 \times \log _{10}(d)+18.3 \times N_{\text {floor }}^{\left(\frac{N_{\text {floor }^{+2}}}{N_{\text {floor }}+1}-0.46\right)}
$$

where $N_{\text {floor }}$ is the number of floors traversed by the transmitted rays. When both the transmitter and receiver are in the same room, $N_{\text {floor }}=1$ and (6) reduces to:

$$
P L(d B)=37+30 \times \log _{10}(d)+18.3
$$

These two models introduced for the path loss modeling in an indoor transmission medium are both widely accepted by the related scientific bodies. However, for the MW-WPT purpose, equation (1) should be preferred since it has frequency component inside, and the power loss factor is higher, as expected for indoor transmission medium.

\subsection{The Use of Narrow-Beam Directional Antenna}

When wide-beam antennas (Omni-directional or Omni-horizontal) are used both at the transmitter and at the receiver, since the transmitted power will scatter $360^{\circ}$ horizontally and the receiver will receive only a portion of the transmitted signal, which can be very small. At the same time, wide-beam antennas improve the device mobility through the transmission medium. When however, highly directional antennas are used, the receiver will be unable to collect some of the multipath components which are outside of its aperture. In general term, directional antennae help to improve path loss by directing the transmitted signal energy in the desired direction. However, in the case of MW-WPT, there is additional gain obtained by 
either eliminating the multipath components or reducing their powers in such a way that:

- The power of the first order reflections will be diminished by the directional antennae, hence, they will not contribute significantly to the amount of collected power;

- The rays that reflect from the obstacles near the antenna will originate from low powered side-lobes, hence their contribution will be small;

- The rays that originate due to second, third or higher order reflections will have very low reflected powers and hence very small contributions;

Hence, due to the reasons stated above, the major contribution to the collected power at the receiver is due to the LOS component.

\subsection{The Rician K-Factor Effect}

In this study, we are using Rician and Rayleigh fading models. Rician fading model is for the case with LOS and Rayleigh is considered for the Non-LOS situations. The Rician fading channel can be easily defined by two specific parameters namely $K$ and $\Omega$. The K-factor in the Rician fading delivers an indication of link quality of the system. Fading will diffuse the signal in a transmission medium. These diffused signals play an important role in the Rician distribution model. Generally the Kfactor parameter represents the ratio between the power of LOS and the power of diffused component. A simple definition of $\mathrm{K}$-factor is presented in (8):

$$
K \text {-factor }=\frac{\text { Power of LOS component }}{\text { Power of diffused component }}
$$


And $\Omega$ is the total power of LOS and diffused component. The K-factor is a statistical explanation in the Rician fading model which differs from medium to medium, experiment to experiment. The effect of $\mathrm{K}$-factor is also examined in the simulation for this study. 


\section{Chapter 4}

\section{MICROWAVE WIRELESS POWER TRANSIMISSION SYSTEM MODELING AND SIMULATION}

System modeling and simulations using SIMULINK is performed in order to validate the MW-WPT in an indoor transmission medium and assess transmission efficiency under various conditions. Checking for the best frequency is one of the most important interests in this work. Comparing the efficiency of different operating frequencies clarify the best choice for MW-WPT system. Also trying different pulse shaping filters indicates what kind of filters should be appropriated for the best system efficiency. Different situations like an always available LOS and a non-LOS (NLOS) condition are considered in the simulation to examine the influence of each state. Use of different transmitter-receiver antenna gains and signal directionality conditions which results in changes in the Rician K-factor, is specified in the test as well. The system setup for simulations is shown in Figure 2.4, where the specific module parameters will be described before each simulation is presented. The data source is simply a train of random data samples $( \pm 1)$, which is made by the pulse shaping filter and then carrier modulation will adjust the transmission frequency to fit the channel conditions and maximize MW-WPT system efficiency. The indoor wireless multipath propagation medium is either a multipath Rayleigh fading channel when a LOS is not available or a multipath Rician fading channel when a LOS is available. The rectenna will simply be a planar microwave antenna with a desired 
gain preceded by a low cut off rectifier diode such as a Schottky Diode. The power collector circuit can be a simple capacitor-diode bank designed as a voltage multiplier as shown in [23]. The block diagram of the simulation program is presented in Figure 4.1.

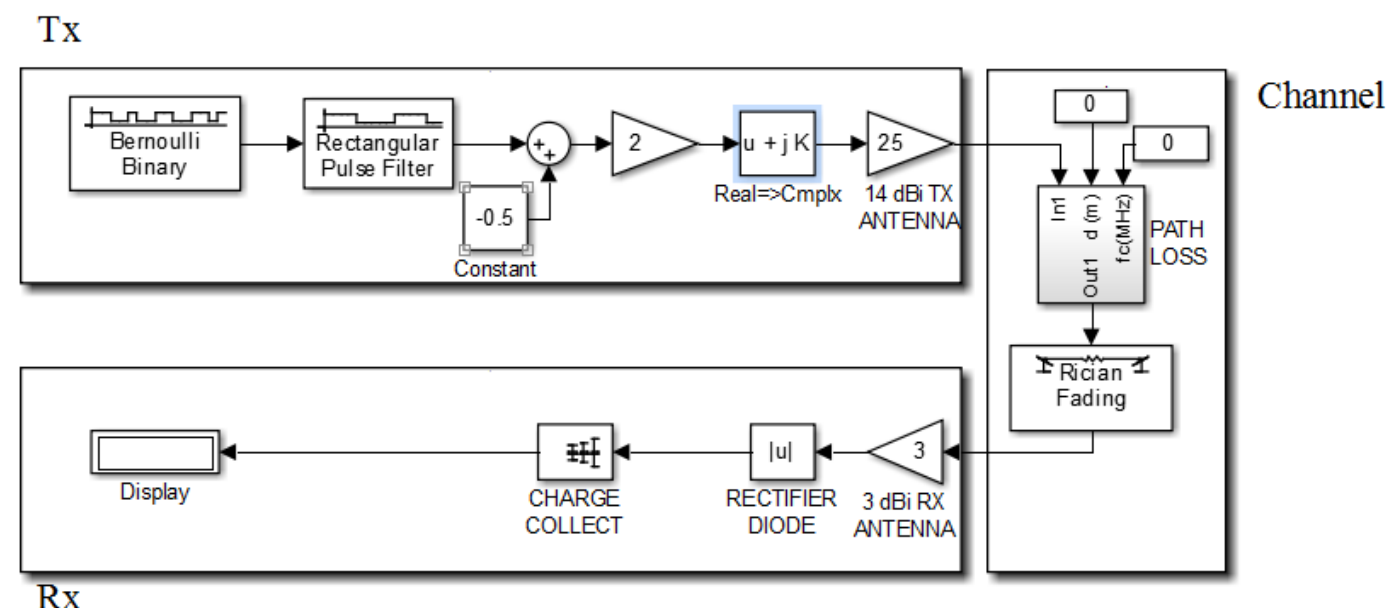

Figure 4.1: System simulation block diagram for rectangular pulse shaping

The Bernoulli binary block in Figure 4.1 is used to produce zeroes and ones. These values will then followed by a pulse shaping filter. The block diagram presented here is for the rectangular pulse shaping test. Later on, to check the result of different pulse shaping, the rectangular pulse shaping block will change into raised cosine pulse shaping filter. After pulse shaping, resulted values are sent to the transmitter antenna with a $14 \mathrm{~dB}$ gain. The channel block contains a path loss block and a fading model block. The path loss block is set using equation (1) and receives distance from the transmitter antenna and the carrier frequency as input data. The result will then go through the fading model block diagram. The fading model block diagram is Rician fading model for LOS case and Rayleigh fading model for Non-LOS 
situation. A $3 \mathrm{~dB}$ gain receiver antenna block is then placed in the receiver part. The gain of the receiver antenna is assumed less than the transmitter antenna because increasing the gain of receiver antenna will increase the actual size of the antenna. In the case we are working on, a huge antenna is not useful at the receiver part as far as the receiver should be easy to carry by the user. A rectifier diode is used after the receiver antenna to rectify the received power and a charge collector block is placed in order to sum up the total power at the receiver. A display block is finally set to show the received power of the system.

Initial simulations are carried out to estimate the average received power at distances varying from 1 to 10 meters. The transmitted power at the transmitter antenna is 1 watt. A basic rectangular pulse shaped data is transmitted over various microwave carrier frequencies to determine the amount of received power at the receiver. The results are presented in Figure 4.2 where the $P_{R x}$ axis is the received power in watt and the $d$ axis is the distance between transmitter and receiver antennas in meter. 


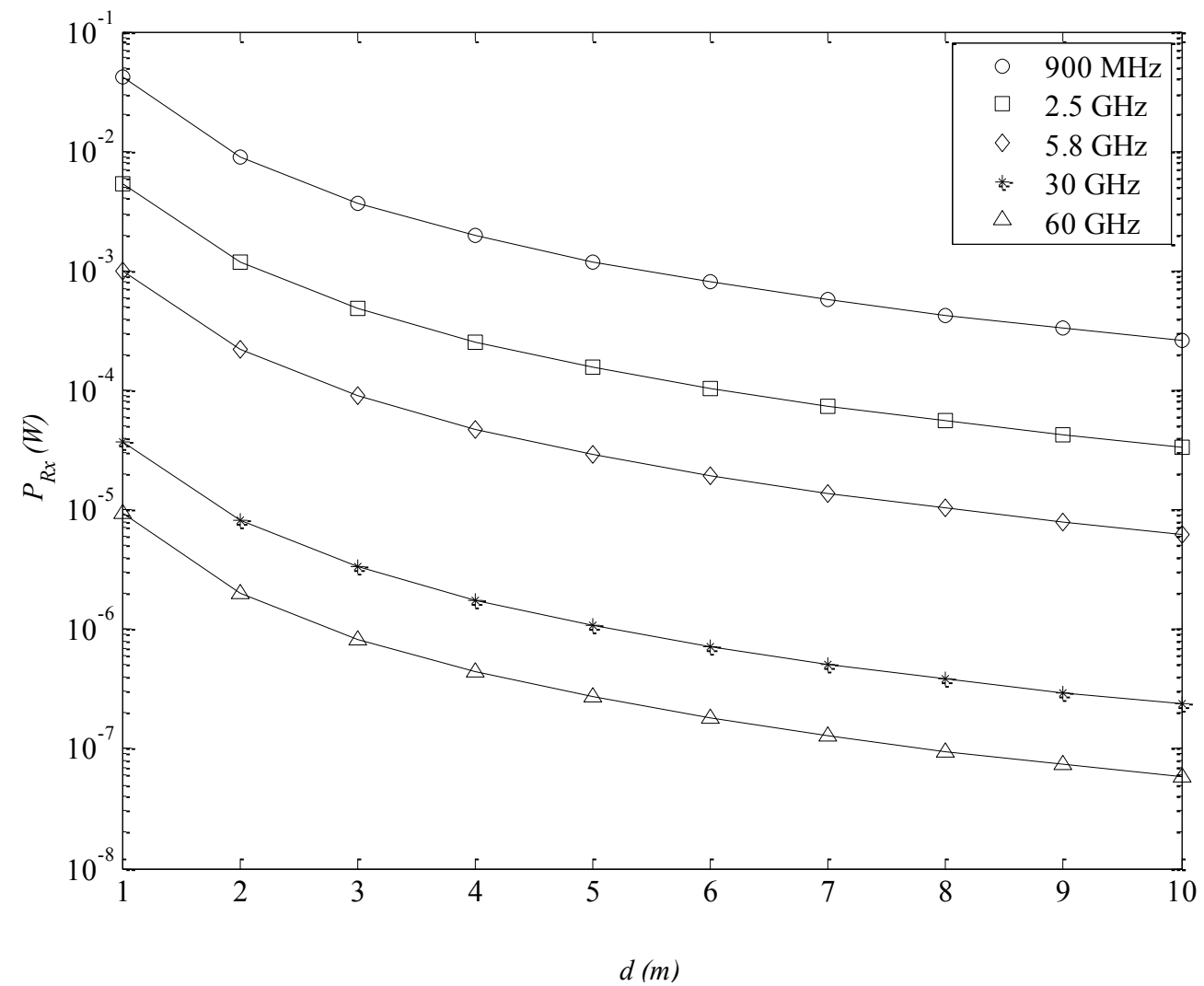

Figure 4.2: $P_{R x}$ versus distance $(d)$ graph for different frequencies of operation for basic rectangular pulse shape

The results indicate that the received power, as it is expected, moderately decreases as the distance between transmitter and receiver increases. Figure 4.2 also specifies that the received power decreases as the operating frequency of the system increase.

Afterward, other simulations were also took placed in order to identify the relative received power using raised cosine pulse shaping, transmitted over various microwave carrier frequencies. So as to clarify the comparison between these two different pulse shaping filters, both results are presented in the same graph in Figure 4.3. 


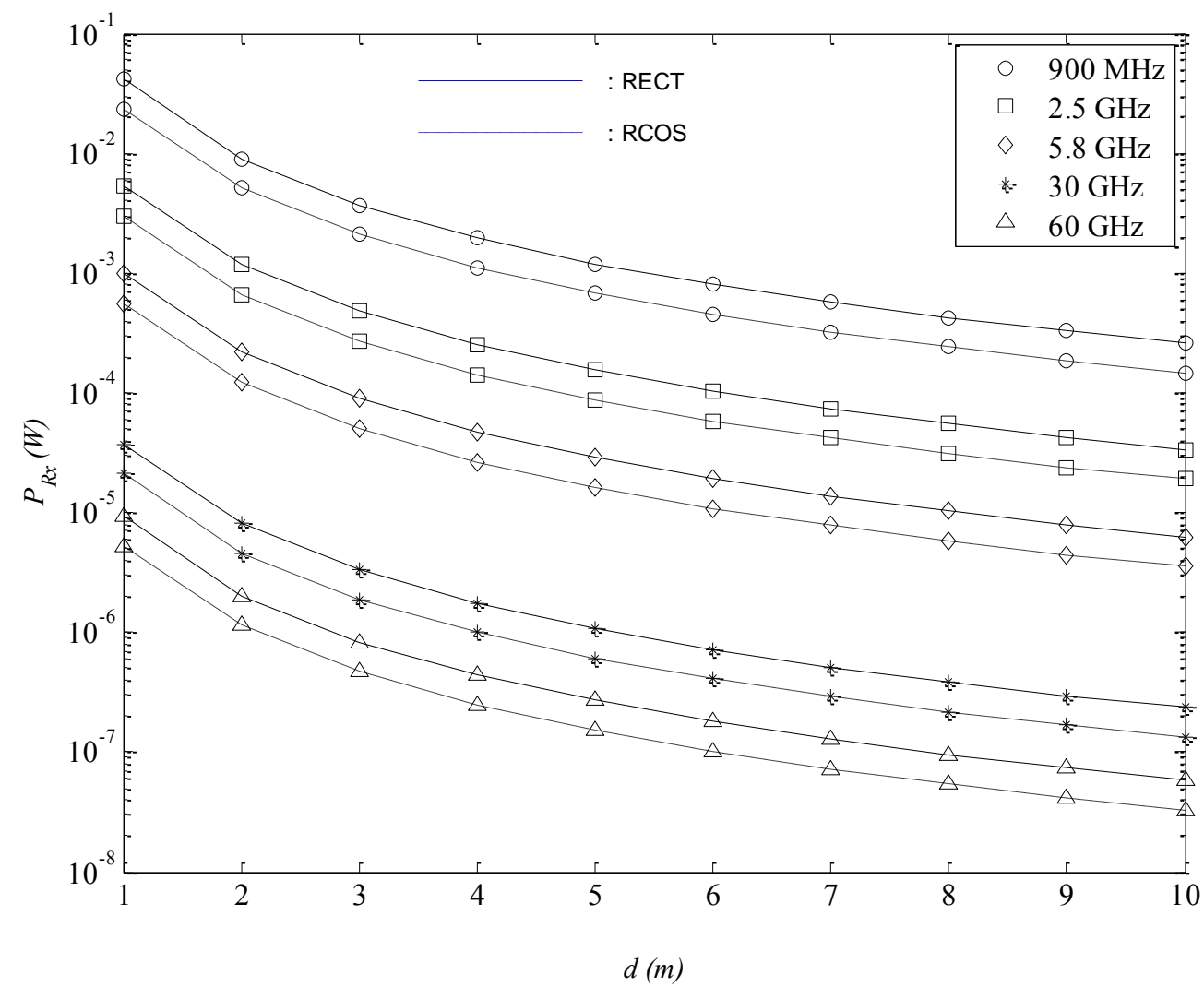

Figure 4.3: $P_{R x}$ versus distance $(d)$ graph for different frequencies of operation comparison between raised cosine pulse shape and rectangular pulse shape

Figure 4.3 shows that the efficiency of the system using raised cosine pulse shaping filter is barely less than same simulation using rectangular pulse shaping filter. Pulse shaping is expected to change the transmitted power and also with the price of increasing interference power for the devices using neighboring frequencies on the spectrum. Simulations are performed in order to estimate the amount of decrease in the received power by using a band limited pulse shape such as raised cosine.

The rectangular pulse shaping is depicted to show a better performance than the raised cosine pulse-shaping technique applied to MW-WPT. The results show that there is a slight loss in the received power level by using raised cosine pulse shape 
compared with the rectangular pulse shape. This is acceptable if limiting interference to the devices using neighboring frequencies is strictly necessary. Otherwise, rectangular pulse shapes can be preferred.

The total efficiency percentage of the system using rectangular pulse shape filter is presented in Figure 4.4 in order to illuminate the transmitted power over received power ratio.

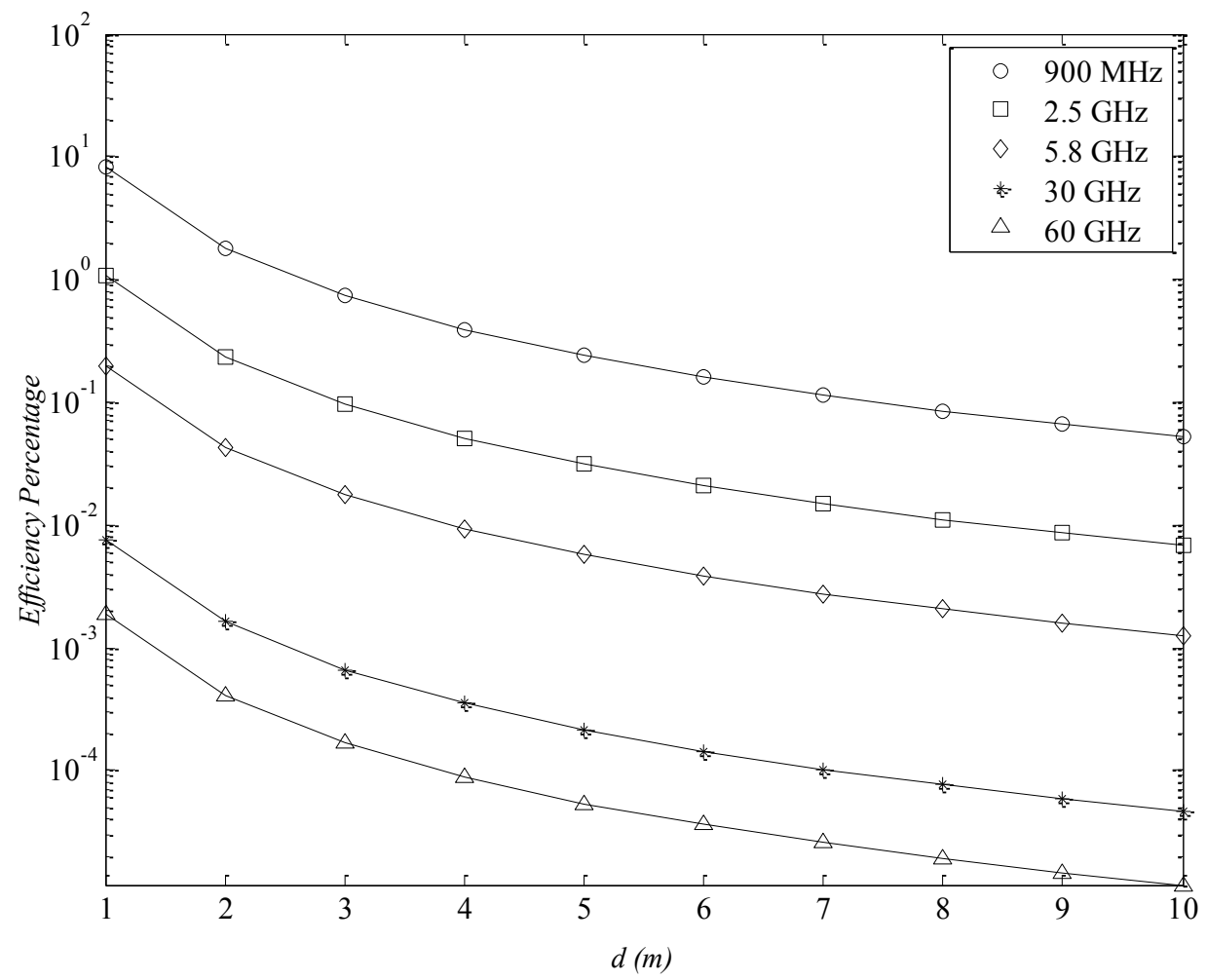

Figure 4.4: MW-WPT system efficiency percentage using rectangular pulse shaping filter

Based on the results from Figure $4.4,900 \mathrm{MHz}$ is the most efficient carrier frequency for the MW-WPT system. Although the received power is important and the 
efficiency of the system depends on that, there is a problem with choosing $900 \mathrm{MHz}$ frequency as the best choice. The $900 \mathrm{MHz}$ carrier frequency is reserved for the telecommunication protocols like radio and television stations. Therefore, using this frequency may bring interfering problems with the communication systems which are using neighboring signal frequencies. Considering all these problems, $2.5 \mathrm{GHz}$ is the best carrier frequency choice for the MW-WPT system efficiency.

According to the results of the simulations, comparing between rectangular pulse shaping and raised cosine pulse shaping, rectangular pulse shaping for the transmitted signal is accepted as the best solution towards the best efficiency of the MW-WPT system. In search for the best technology to optimize the MW-WPT system, a thorough study have also been done to find out if the duty-cycle of the rectangular pulse train has any effect on the system efficiency. It can easily be shown that, due to the use of antipodal signaling $( \pm 1)$, the received power will always be the same. Therefore, the duty-cycle of the rectangular pulse train has no effect on transmission efficiency.

The MW-WPT system should be so designed that there is always a LOS between the transmitter and the receiver. However, there might be instances where the LOS could be blocked for periods of time which may have substantial effects on the transmission. The indoor propagation characteristics may vary due to movement of persons and objects in the transmission environment as well. Nevertheless these changes are not so effective and can be preserved as a time invariant random variable. Simulations have been done in order to test these situations and the results 
are presented for both LOS and NLOS cases in Figure 4.5 for the rectangular shaped pulses.

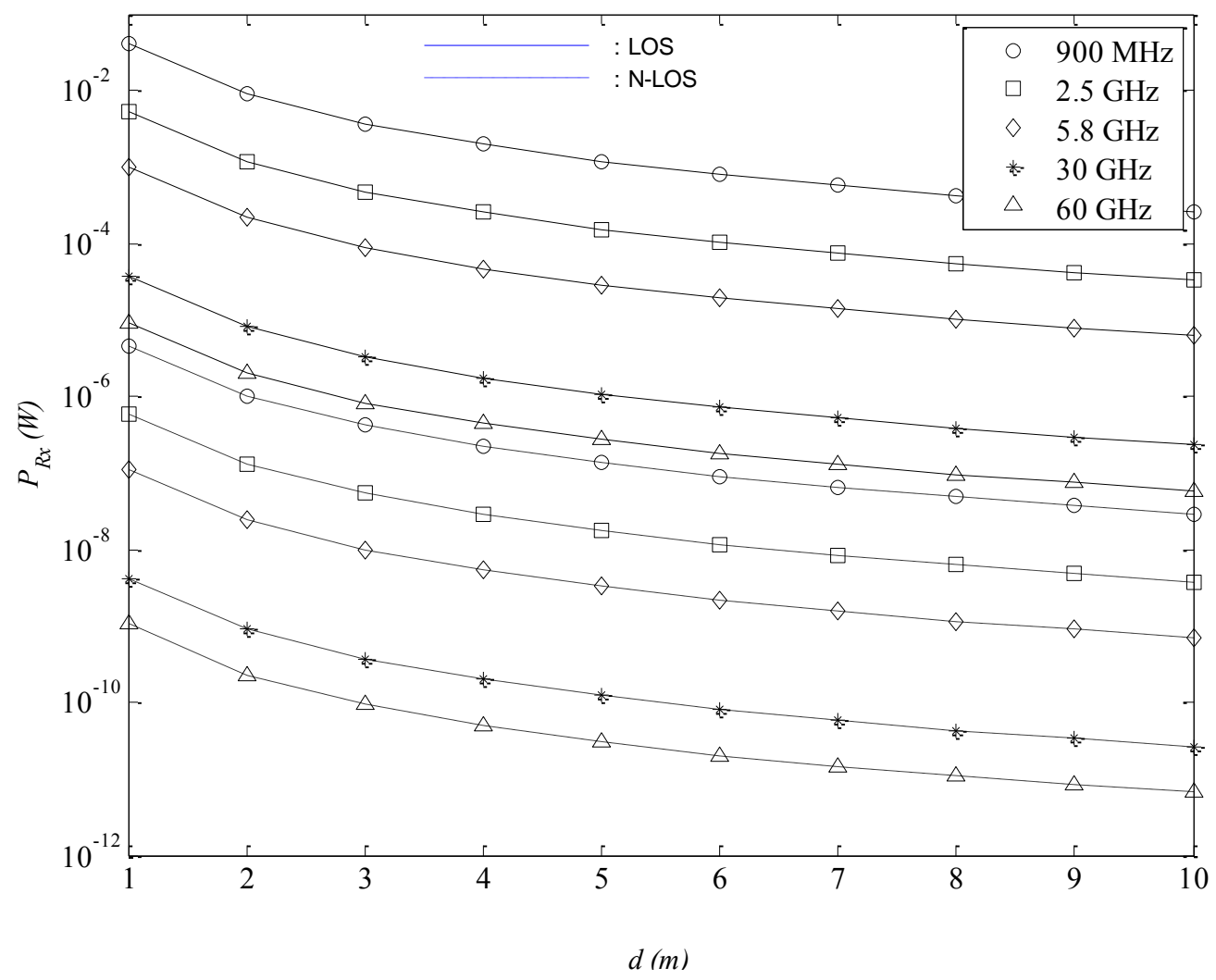

Figure 4.5: $P_{R x}$ versus distance $(d)$ for LOS and non-LOS (NLOS) cases of rectangular pulse shape

Figure 4.5 states that in case there is no available LOS, the amount of received power to the rectenna will sharply decrease. As it is obvious from the figure above, in NLOS case the received power of a $900 \mathrm{MHz}$ carrier frequency is even less than a 60 $\mathrm{GHz}$ transmission system over an existing LOS situation. Thus, the loss of LOS in the MW-WPT system may cause a significant reduction in the system efficiency. 
Increasing the transmitter and receiver antenna gains clearly show a drastic improvement in the system efficiency. By using high gain antennas, the effective aperture of the antenna will increase additionally. Based on the equation (9):

$$
G=\frac{4 \pi A_{e}}{\lambda^{2}}
$$

where $G$ is the antenna gain, $\lambda$ is the wave length in meters and $A_{e}$ is the effective aperture that depends on the physical size of the antenna. Hence, to increase the gain of the antenna with a constant wave length, the physical size of the antenna should become larger. Increasing the gain of the transmitter and receiver antennas improves the system efficiency to a level where MW-WPT technique becomes sufficiently high in order to charge such devices as smart phone batteries in practice, yet the size of the transmitter and receiver antennas are important and are not expected to become extremely huge. The directional antennas on the other hand, can easily increase the total efficiency of the system by eliminating scattering of the multipath components in the transmission medium.

To find the best solution to improve the efficiency of the MW-WPT system in an indoor environment, the effect of Rician K-factor should also be investigated. Simulations are done for the $2.5 \mathrm{GHz}$ operation frequency considering varying $\mathrm{K}$ factors. Result of the simulation is shown in Figure 4.6. 


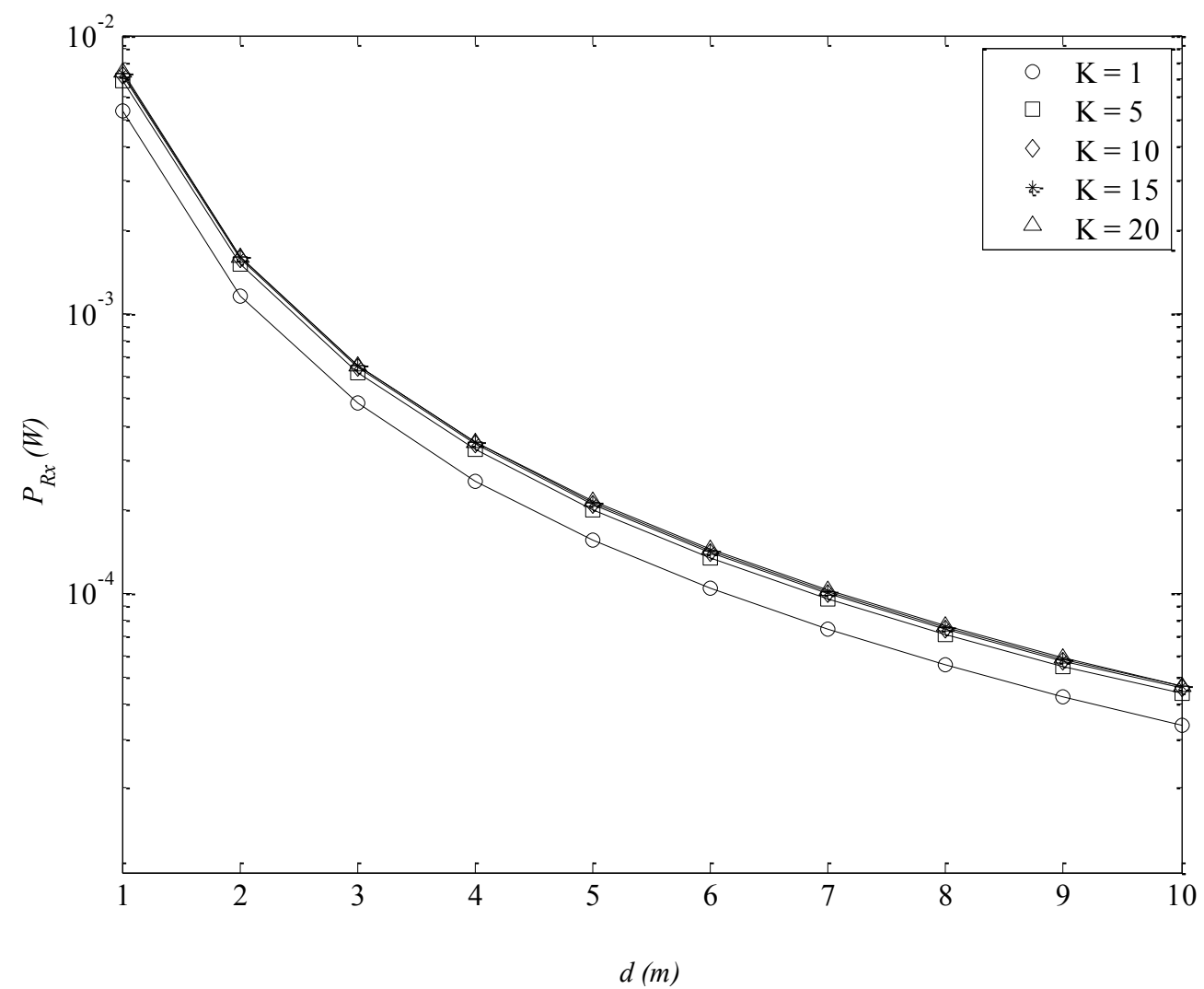

Figure 4.6: $P_{R x}$ versus distance $(d)$ for different Rician K-Factors of rectangular pulse shape with $2.5 \mathrm{GHz}$ carrier frequency

It can be judged from Figure 4.6 that the Rician K-factor, hence the strengths of reflected rays relative to the LOS have insignificant changes on MW-WPT efficiency. 


\section{Chapter 5}

\section{CONCLUSION AND RECOMMENDATIONS FOR FURTHER STUDIES}

\subsection{Conclusion}

Due to the need to supply increasing amounts of power in order to implement sophisticated functions such as video processing and rendering and wideband data communication, there is an urgent need to charge batteries of electronics communication devices such as smart phones very often. Therefore, providing wireless charging points suitable for all sorts of electronics device batteries can be a smart solution. This work has been carried out in order to prove by simulations that design and implementation of such a system is possible.

The simulation results have shown that, rectangular pulses can carry more power than raised-cosine pulses. It was also concluded that duty cycle of the rectangular pulses has no effect on transmission efficiency. It was also clear from the results that, without using a highly directional antenna, the received signal power is not sufficient to charge a typical smart phone battery. The availability of a strong LOS has been shown to improve system efficiency significantly.

It can also be concluded that, by filtering, a proportion of the transmitted power is eliminated by the transmitter. But filtering could be strictly necessary in order to 
avoid co-channel interference to devices using the neighboring frequencies in electronic communication device crowded environments. Filtering, by such as raised-cosine filter, on the other hand, is shown to reduce MW-WPT efficiency since a portion of the received power is eliminated by the filter.

\subsection{Recommendations for Future Works}

Investigation of the efficiency improvement by various diversity techniques is a subject for further study. The Wireless Power Transmission (WPT) work at lower frequencies such as $100 \mathrm{MHz}$ and lower is also subject for further study. Finding a lower frequency which is not reserved for any communication systems can be suggested as an appropriated solution to the problem. Meanwhile, working on lower frequencies will increase the size of transmitter and receiver antennas at the same time. Therefore, finding the best balance between the frequency and the antenna size can give the best result for the MW-WPT system efficiency. 


\section{REFERENCES}

[1] T. P. Hughes, Networks Of Power: Electrification in Western Society 18801930, London: Johns Hopkins University Press, 1983.

[2] N. Tesla, Apparatus for transmitting electrical energy, USA Patent, 1914.

[3] NASA, Beamed laser power for UAVs, Dryden Flight Research Center, 2003.

[4] Philippe Basset, "Complete system for wireless powering and remote control of electrostatic actuators by inductive coupling," vol. 12, no. 1, 2007.

[5] J. Gao, "Traveling magnetic field for homogeneous wireless power transmission," vol. 22, no. 1, 2007.

[6] Zhen Ning Low, "Design and test of a high-power high-efficiency loosely coupled planar wireless power transfer system," vol. 56, no. 5, 2009.

[7] W. C. Brown, "The history of power transmission by radio waves," vol. 32, no. 9, 1984.

[8] R. M. Dickinson, "Performance of e high-power 2.388-GHz receiving array in wireless power transmission over $1.54 \mathrm{~km}, " 1976$.

[9] P. K. A. J. T. Cha, "Millimeter wave technology for space power beaming," vol. 40, no. 6, 1992.

[10] McSpadden, "Theoretical and experimental investigation of a rectenna element for microwave power transmission," vol. 40, no. 12, 1992.

[11] Y. Fujino, "11. Y. Fujino, T. Ito, M. Fujita, N. Kaya, H. MatsumotoA driving 
test of a small dc motor with a rectenna array," Vols. E77-B, no. 4, 1994.

[12] T. Yoo, "35 GHz rectenna implemented with a patch and a microstrip dipole antenna," 1992.

[13] S. Larry W. Epp, "A Compact Dual-Polarized 8.51-GHz Rectenna for HighVoltage (50 V) Actuator Applications," vol. 48, no. 1, 2000.

[14] K. Chang, "Theoretical and Experimental Development of 10 and $35 \mathrm{GHz}$ Rectennas," vol. 40, no. 6, 1992.

[15] W.C. Brown, "An Experimental Microwave-Powered Helicopter," vol. 13, no. $5,1965$.

[16] P. J. R. Smith, "WREL," 2011. [Online]. Available: http://sensor.cs.washington.edu/WREL.html.

[17] I. E. Commission, IEC 60825 Safety of laser products,Part 1: Equipment classification and requirements, 2007.

[18] D. G. Aakash Sahai, "Optical Wireless Power Transmission at Long Wavelengths," 2011.

[19] J. Doutreloigne, "Fully Integrated Dickson Charge Pumps with Optimized Power Efficiency," vol. 2, 2010.

[20] D. C. C. William C. Jakes, Microwave Mobile Communications, Wiley-IEEE Press, 1994.

[21] I. T. U. (ITU), Propagation Data and Prediction Methods for the Planning of Indoor Radiocommunication Systems and Radio Local Area Networks in the Frequency Range $900 \mathrm{MHz}$ to 100GHz, ITU-R P.1238-7, Geneva: ITU-R 
Radiocommunication Sector of ITU, 02/2012.

[22] A. F.Molisch, Wireless Communications, John Wiley \& Sons, Ltd, 2005.

[23] Vlad Marian, "Wireless Energy Transfer Using Zero Bias Schottky Diodes Rectenna Structures," Advanced Materials Research, Trans Tech Publications, pp. 449-452, 2011. 